\title{
Double-Framed Soft Deductive System of Subtraction Algebras
}

Chul Hwan Park

School of Mechanical Engineering, Ulsan College, Ulsan, Korea

\section{]jfis}

Received: Aug. 30, 2018

cCThis is an Open Access article distributed under the terms of the Creative Commons Attribution Non-Commercial License (http://creativecommons.org/licenses/ by-nc/3.0// which permits unrestricted noncommercial use, distribution, and reproduction in any medium, provided the original work is properly cited.

\begin{abstract}
The notion of double-framed soft deductive system in subtraction algebras is introduced, and several properties are investigated. Conditions for a double-framed soft set to be a double-framed soft deductive system are provided. Characterizations of a double-framed soft deductive system are established.
\end{abstract}

Keywords: Subtraction algebra, Soft set, Double-framed soft set, Double-framed soft deductive system

\section{Introduction}

Zadeh [1] introduced the concept of fuzzy set as a new mathematical tool for dealing with uncertainties and so many researchers were conducted on the generalizations of the notion of fuzzy sets. Various theories have their own difficulties which are pointed out in [2]. To overcome these difficulties, Molodtsov [2] introduced the concept of soft set as a new mathematical tool for dealing with uncertainties that is free from the difficulties that have troubled the usual theoretical approaches. Maji et al. [3, 4] also studied several operations on the theory of soft sets and described the application of soft set theory to a decision-making problem. Aktas and Cagman [5] studied the basic concepts of soft set theory and discussed the notion of soft groups. Jun and Park [6] studied applications of soft sets in ideal theory of $B C K / B C I$-algebras. We refer the reader to the papers [7-15] for further information regarding algebraic structures/properties of soft set theory.

Schein [16] have considered systems of the form $(\Phi ; \circ, \backslash)$, where $\Phi$ is a set of functions closed under the composition " $\circ$ " of functions (and hence $(\Phi ; \circ)$ is a function semigroup) and the set theoretic subtraction "\" (and hence $(\Phi ; \backslash)$ is a subtraction algebra in the sense of [17]). Jun and Kim [18] discussed ideal theory of subtraction algebras.

In this paper we introduce a notion of double-framed soft deductive system in a subtraction algebra, and study some properties of them. We give conditions for a double-framed soft set to be a double-framed soft deductive system and establish characterizations of a double-framed soft deductive system

\section{Preliminaries}

A subtraction algebra is a system $(X ;-)$ with a single binary operation "-" that satisfies the following identities: for any $x, y, z \in X$, 


$$
\begin{aligned}
& x-(y-x)=x \\
& x-(x-y)=y-(y-x) \\
& (x-y)-z=(x-z)-y
\end{aligned}
$$

The last identity permits us to omit parentheses in expressions of the form $(x-y)-z$. For any $a, b \in X$, we define an order relation $\leq$ on $X: a \leq b \Leftrightarrow a-b=0$, where $0=a-a$ is an element that does not depend on the choice of $a \in X$. The ordered set $(X ; \leq)$ is a semi-Boolean algebra in the sense of [17], that is, it is a meet semilattice with zero 0 in which every interval $[0, a]$ is a Boolean algebra with respect to the induced order. Here $a \wedge b=a-(a-b)$; the complement of an element $b \in[0, a]$ is $a-b$; and if $b, c \in[0, a]$, then

$$
\begin{aligned}
b \vee c & =\left(b^{\prime} \wedge c^{\prime}\right)^{\prime}=a-((a-b) \wedge(a-c)) \\
& =a-((a-b)-((a-b)-(a-c))) .
\end{aligned}
$$

In a subtraction algebra, the following are true (see [18]):

$$
\begin{aligned}
& (x-y)-y=x-y . \\
& x-0=x \text { and } 0-x=0 . \\
& (x-y)-x=0 . \\
& x-(x-y) \leq y . \\
& (x-y)-(y-x)=x-y . \\
& x-(x-(x-y))=x-y . \\
& (x-y)-(z-y) \leq x-z . \\
& x \leq y \text { if and ony if } x=y-w \text { for some } w \in X . \\
& x \leq y \text { implies } x-z \leq y-z \text { and } z-y \leq z-x \\
& \text { for all } z \in X . \\
& x, y \leq z \text { implies } x-y=x \wedge(z-y) . \\
& (x \wedge y)-(x \wedge z) \leq x \wedge(y-z) .
\end{aligned}
$$

Proposition 2.1 ( $[18])$. Let $X$ be a subtraction algebra and let $x, y \in X$. If $w \in X$ is an upper bound for $x$ and $y$, then the element

$$
x \vee y:=w-((w-y)-x)
$$

is a least upper bound for $x$ and $y$.

Definition 2.2 ([18]). A nonempty subset $S$ of $X$ is called a subalgebra of $X$ if it satisfies :

$$
(\forall x, y \in S)(x-y \in S)
$$

Definition 2.3. A nonempty subset $D$ of $X$ is called a deductive system of $X$ (it is called an ideal of $X$ in [18]) if it satisfies:
(1) $0 \in D$,

(2) $(\forall x \in X)(\forall y \in D)(x-y \in D \Rightarrow x \in D)$.

Molodtsov [2] defined the soft set in the following way: Let $U$ be an initial universe set and $E$ be a set of parameters. Let $\mathscr{P}(U)$ denotes the power set of $U$ and $A, B, C, \cdots \subseteq E$.

Definition 2.4 ( [2]). A pair $(\tilde{\alpha}, A)$ is called a soft set over $U$, where $\tilde{\alpha}$ is a mapping given by

$$
\tilde{\alpha}: A \rightarrow \mathscr{P}(U)
$$

In other words, a soft set over $U$ is a parameterized family of subsets of the universe $U$. For $\varepsilon \in A, \tilde{\alpha}(\varepsilon)$ may be considered as the set of $\varepsilon$-approximate elements of the soft set $(\tilde{\alpha}, A)$. Clearly, a soft set is not a set. For illustration, Molodtsov [2] considered several examples in [2].

Definition 2.5 ( $[10])$. A double-framed pair $\langle(\tilde{\alpha}, \tilde{\beta}) ; A\rangle$ is called a double-framed soft set of $A$ over $U$, where $\tilde{\alpha}$ and $\tilde{\beta}$ are mappings from $A$ to $\mathscr{P}(U)$.

\section{Double-Framed Soft Deductive Systems}

In what follows, we take $E=X$, as a set of parameters, which is a subtraction algebra and $A, B, C, \cdots$ be subalgebras of $X$ unless otherwise specified.

Definition 3.1. A double-framed soft $\langle(\tilde{\alpha}, \tilde{\beta}) ; X\rangle$ of $X$ is called a double frame soft deductive system set of $X$ over $U$ if the following conditions are true:

$$
\begin{array}{r}
(\forall x \in X)(\tilde{\alpha}(0) \supseteq \tilde{\alpha}(x), \tilde{\beta}(0) \subseteq \tilde{\beta}(x)), \\
(\forall x, y \in X)(\tilde{\alpha}(x) \supseteq \tilde{\alpha}(x-y) \cap \tilde{\alpha}(y), \\
\tilde{\beta}(x) \subseteq \tilde{\beta}(x-y) \cup \tilde{\beta}(y)),
\end{array}
$$

Example 3.2. Consider a subtraction algebra

$$
X=\left\{0, e_{1}, e_{2}, e_{3}, e_{4}\right\}
$$

with the following Cayley table [19]:

\begin{tabular}{c|ccccc}
- & 0 & $e_{1}$ & $e_{2}$ & $e_{3}$ & $e_{4}$ \\
\hline 0 & 0 & 0 & 0 & 0 & 0 \\
$e_{1}$ & $e_{1}$ & 0 & $e_{1}$ & 0 & $e_{1}$ \\
$e_{2}$ & $e_{2}$ & $e_{2}$ & 0 & 0 & $e_{2}$ \\
$e_{3}$ & $e_{3}$ & $e_{2}$ & $e_{1}$ & 0 & $e_{3}$ \\
$e_{4}$ & $e_{4}$ & $e_{4}$ & $e_{4}$ & $e_{4}$ & 0
\end{tabular}


For $A=X$ and $U=\left\{h_{1}, h_{2}, h_{3}, h_{4}, h_{5}, h_{6}\right\}$. We define a double-framed soft set $\langle(\tilde{\alpha}, \tilde{\beta}) ; A\rangle$ over $U$ as follows:

$$
\tilde{\alpha}: A \rightarrow \mathscr{P}(U), x \mapsto \begin{cases}\left\{h_{1}, h_{3}, h_{4}, h_{5}\right\} & \text { if } x=0, \\ \left\{h_{1}, h_{3}, h_{4}, h_{5}\right\} & \text { if } x=e_{1}, \\ \left\{h_{3}, h_{4}, h_{5}\right\} & \text { if } x=e_{2}, \\ \left\{h_{3}, h_{4}, h_{5}\right\} & \text { if } x=e_{3}, \\ \left\{h_{1}, h_{3}, h_{4}, h_{5}\right\} & \text { if } x=e_{4},\end{cases}
$$

and

$$
\tilde{\beta}: A \rightarrow \mathscr{P}(U), x \mapsto \begin{cases}\left\{h_{1}, h_{3}\right\} & \text { if } x=0, \\ \left\{h_{1}, h_{3}\right\} & \text { if } x=e_{1}, \\ \left\{h_{1}, h_{3}, h_{4}\right\} & \text { if } x=e_{2} \\ \left\{h_{1}, h_{3}, h_{4}\right\} & \text { if } x=e_{3}, \\ \left\{h_{1}, h_{3}\right\} & \text { if } x=e_{4} .\end{cases}
$$

It is routine to verify that $\langle(\tilde{\alpha}, \tilde{\beta}) ; A\rangle$ is a double-framed soft deductive system of $X$ over $U$.

Proposition 3.3. Every double-framed soft deductive system $\langle(\tilde{\alpha}, \tilde{\beta}) ; X\rangle$ of $X$ over $U$ satisfies:

$$
(\forall x, y \in X)(x \leq y \Rightarrow \tilde{\alpha}(x) \supseteq \tilde{\alpha}(y), \tilde{\beta}(x) \subseteq \tilde{\beta}(y)) .
$$

Proof. Let $x, y \in X$ be such that $x \leq y$. Then $x-y=0$, and so

$$
\begin{aligned}
& \tilde{\alpha}(x) \supseteq \tilde{\alpha}(x-y) \cap \tilde{\alpha}(y)=\tilde{\alpha}(0) \cap \tilde{\alpha}(y)=\tilde{\alpha}(y), \\
& \tilde{\beta}(x) \subseteq \tilde{\beta}(x-y) \cup \tilde{\beta}(y)=\tilde{\beta}(0) \cup \tilde{\beta}(y)=\tilde{\beta}(y) .
\end{aligned}
$$

Proposition 3.4. Every double-framed soft deductive system $\langle(\tilde{\alpha}, \tilde{\beta}) ; X\rangle$ of $X$ over $U$ satisfies:

$$
(\forall x, y, z \in X)\left(\begin{array}{l}
\tilde{\alpha}(x-z) \supseteq \tilde{\alpha}((x-y)-z) \cap \tilde{\alpha}(y), \\
\tilde{\beta}(x-z) \subseteq \tilde{\beta}((x-y)-z) \cup \tilde{\beta}(y) .
\end{array}\right)
$$

Proof. Combining (S3) and Definition 3.1(c2), we get

$$
\begin{aligned}
\tilde{\alpha}(x-z) & \supseteq \tilde{\alpha}((x-z)-y) \cap \tilde{\alpha}(y) \\
& =\tilde{\alpha}((x-y)-z) \cap \tilde{\alpha}(y),
\end{aligned}
$$

and

$$
\begin{aligned}
\tilde{\beta}(x-z) & \subseteq \tilde{\beta}((x-z)-y) \cup \tilde{\beta}(y) \\
& =\tilde{\beta}((x-y)-z) \cup \tilde{\beta}(y)
\end{aligned}
$$

for all $x, y, z \in X$.

We give conditions for a double-framed soft set to be a double-framed soft deductive system .

Theorem 3.5. If double-framed soft set $\langle(\tilde{\alpha}, \tilde{\beta}) ; X\rangle$ of $X$ over $U$ satisfying (c1) and $\{2$, then $\langle(\tilde{\alpha}, \tilde{\beta}) ; X\rangle$ is a double-framed soft deductive system of $X$ over $U$.

Proof. Taking $z=0$ in (2) and using (a2), we get

$$
\begin{aligned}
\tilde{\alpha}(x) & =\tilde{\alpha}(x-0) \supseteq \tilde{\alpha}((x-y)-0) \cap \tilde{\alpha}(y) \\
& =\tilde{\alpha}(x-y) \cap \tilde{\alpha}(y)
\end{aligned}
$$

and

$$
\begin{aligned}
\tilde{\beta}(x) & =\tilde{\beta}(x-0) \subseteq \tilde{\beta}((x-y)-0) \cup \tilde{\beta}(y) \\
& =\tilde{\beta}(x-y) \cup \tilde{\beta}(y)
\end{aligned}
$$

for all $x, y \in X$. Hence $\langle(\tilde{\alpha}, \tilde{\beta}) ; X\rangle$ is a double-framed soft deductive system of $X$ over $U$.

Corollary 3.6. Let $\langle(\tilde{\alpha}, \tilde{\beta}) ; X\rangle$ be a double-framed soft set of $X$ over $U$. Then $\langle(\tilde{\alpha}, \tilde{\beta}) ; X\rangle$ is a double-framed soft deductive system of $X$ over $U$ if and only if it satisfies conditions (c1) and (2).

The following is a characterization of a double-framed soft deductive system of $X$ over $U$.

Theorem 3.7. Let $\langle(\tilde{\alpha}, \tilde{\beta}) ; X\rangle$ be a double-framed soft set of $X$ over $U$. Then $\langle(\tilde{\alpha}, \tilde{\beta}) ; X\rangle$ is a double-framed soft deductive system of $X$ over $U$ if and only if it satisfies the following conditions:

$$
\begin{aligned}
& (\forall x, y \in X)(\tilde{\alpha}(x-y) \supseteq \tilde{\alpha}(x), \tilde{\beta}(x-y) \subseteq \tilde{\beta}(x)) \\
& (\forall x, a, b \in X)\left(\begin{array}{l}
\tilde{\alpha}(x-((x-a)-b)) \supseteq \tilde{\alpha}(a) \cap \tilde{\alpha}(b), \\
\tilde{\beta}(x-((x-a)-b)) \subseteq \tilde{\beta}(a) \cup \tilde{\beta}(b)
\end{array}\right) .
\end{aligned}
$$

Proof. Assume that $\langle(\tilde{\alpha}, \tilde{\beta}) ; X\rangle$ is a double-framed soft deductive system of $X$ over $U$. Combining (a3) and Defintion 3.1. we have

$$
\begin{aligned}
\tilde{\alpha}(x-y) & \supseteq \tilde{\alpha}((x-y)-x) \cap \tilde{\alpha}(x)=\tilde{\alpha}(0) \cap \tilde{\alpha}(x) \\
& =\tilde{\alpha}(x),
\end{aligned}
$$

and

$$
\tilde{\beta}(x-y) \subseteq \tilde{\beta}((x-y)-x) \cup \tilde{\beta}(x)=\tilde{\beta}(0) \cup \tilde{\beta}(x)
$$




$$
=\tilde{\beta}(x)
$$

for all $x, y \in X$. Since

$$
(x-((x-a)-b))-a=(x-a)-((x-a)-b) \leq b,
$$

it follows from (1) that

$$
\tilde{\alpha}((x-((x-a)-b))-a) \supseteq \tilde{\alpha}(b),
$$

and

$$
\tilde{\beta}((x-((x-a)-b))-a) \subseteq \tilde{\beta}(b)
$$

so from (c2) that

$$
\begin{aligned}
& \tilde{\alpha}(x-((x-a))-b)) \\
& \supseteq \tilde{\alpha}((x-((x-a)-b))-a) \cap \tilde{\alpha}(a) \\
& \supseteq \tilde{\alpha}(a) \cap \tilde{\alpha}(b),
\end{aligned}
$$

and

$$
\begin{aligned}
& \tilde{\beta}(x-((x-a))-b)) \\
& \subseteq \tilde{\beta}((x-((x-a)-b))-a) \cup \tilde{\beta}(a) \\
& \subseteq \tilde{\beta}(a) \cup \tilde{\beta}(b) .
\end{aligned}
$$

Conversely assume that $\langle(\tilde{\alpha}, \tilde{\beta}) ; X\rangle$ be a double-framed soft set of $X$ over $U$ satisfying conditions (3) and (4). If we take $y=x$ in 3 , then $\tilde{\alpha}(0)=\tilde{\alpha}(x-x) \supseteq \tilde{\alpha}(x)$ and $\tilde{\beta}(0)=$ $\tilde{\beta}(x-x) \subseteq \tilde{\beta}(x)$ for all $x \in X$. By $(4)$, we obtain

$$
\begin{aligned}
\tilde{\alpha}(x) & =\tilde{\alpha}(x-0) \\
& =\tilde{\alpha}(x-((x-y)-(x-y))) \\
& =\tilde{\alpha}(x-((x-(x-y))-y)) \\
& \supseteq \tilde{\alpha}(x-y) \cap \tilde{\alpha}(y),
\end{aligned}
$$

and

$$
\begin{aligned}
\tilde{\beta}(x) & =\tilde{\beta}(x-0) \\
& =\tilde{\beta}(x-((x-y)-(x-y))) \\
& =\tilde{\beta}(x-((x-(x-y))-y)) \\
& \subseteq \tilde{\beta}(x-y) \cup \tilde{\beta}(y)
\end{aligned}
$$

for all $x, y \in X$. Hence $\langle(\tilde{\alpha}, \tilde{\beta}) ; X\rangle$ is a double-framed soft deductive system of $X$ over $U$.

Proposition 3.8. Every double-framed soft deductive system $\langle(\tilde{\alpha}, \tilde{\beta}) ; X\rangle$ of $X$ over $U$ satisfies the following assertion:

$$
(\forall x, y \in X)\left(\exists x \vee y \Rightarrow\left(\begin{array}{c}
\tilde{\alpha}(x \vee y) \supseteq \tilde{\alpha}(x) \cap \tilde{\alpha}(y) \\
\tilde{\beta}(x \vee y) \subseteq \tilde{\beta}(x) \cap \tilde{\beta}(y)
\end{array}\right)\right)
$$

Proof. Suppose there exists $x \vee y$ for $x, y \in X$. Let $w$ be an upper bound of $x$ and $y$. Then $x \vee y=w-((w-y)-x)$ is the least upper bound for $x$ and $y$ (see Proposition 2.1), and so

$$
\tilde{\alpha}(x \vee y)=\tilde{\alpha}(w-((w-y)-x)) \supseteq \tilde{\alpha}(x) \cap \tilde{\alpha}(y),
$$

and

$$
\tilde{\beta}(x \vee y)=\tilde{\beta}(w-((w-y)-x)) \subseteq \tilde{\beta}(x) \cup \tilde{\beta}(y)
$$

by (4). This completes the proof.

Proposition 3.9. Let $\langle(\tilde{\alpha}, \tilde{\beta}) ; X\rangle$ be a double-framed soft of $X$ over $U$. Then $\langle(\tilde{\alpha}, \tilde{\beta}) ; X\rangle$ is a double-framed soft deductive system set of $X$ over $U$ if and only if it satisfies:

$$
(\forall x, y, z \in X)\left(x-y \leq z \Rightarrow\left(\begin{array}{c}
\tilde{\alpha}(x) \supseteq \tilde{\alpha}(y) \cap \tilde{\alpha}(z), \\
\tilde{\beta}(x) \subseteq \tilde{\beta}(y) \cup \tilde{\beta}(z)
\end{array}\right)\right)
$$

Proof. Assume that $\langle(\tilde{\alpha}, \tilde{\beta}) ; X\rangle$ is a double-framed soft of $X$ over $U$ and let $x, y, z \in X$ be such that $x-y \leq z$. Then $\tilde{\alpha}(z) \subseteq \tilde{\alpha}(x-y)$ and $\tilde{\beta}(z) \supseteq \tilde{\beta}(x-y)$ by 11 . It follows from (c2) that

$$
\tilde{\alpha}(x) \supseteq \tilde{\alpha}(x-y) \cap \tilde{\alpha}(y) \supseteq \tilde{\alpha}(y) \cap \tilde{\alpha}(z),
$$

and

$$
\tilde{\beta}(x) \subseteq \tilde{\beta}(x-y) \cup \tilde{\beta}(y) \subseteq \tilde{\beta}(y) \cup \tilde{\beta}(z) .
$$

Conversely suppose that $\langle(\tilde{\alpha}, \tilde{\beta}) ; X\rangle$ satisfies $\sqrt{6}$. Since $0-y \leq y$ for all $y \in X$, we have

$$
\tilde{\alpha}(0) \supseteq \tilde{\alpha}(y) \cap \tilde{\alpha}(y)=\tilde{\alpha}(y),
$$

and

$$
\tilde{\beta}(0) \subseteq \tilde{\beta}(y) \cup \tilde{\beta}(y)=\tilde{\beta}(y)
$$

by (6). Thus (c1) is valid. Since $x-(x-y) \leq y$ for all $x, y \in X$ by (a4), it follows from $(\sqrt{6})$ that $\tilde{\alpha}(x) \supseteq \tilde{\alpha}(x-y) \cap \tilde{\alpha}(y)$ and $\tilde{\beta}(x) \subseteq \tilde{\beta}(x-y) \cup \tilde{\beta}(y)$. Hence $\langle(\tilde{\alpha}, \tilde{\beta}) ; X\rangle$ is a double-framed soft deductive system of $X$ over $U$.

Let $\langle(\tilde{\alpha}, \tilde{\beta}) ; A\rangle$ and $\langle(\tilde{f}, \tilde{g}) ; B\rangle$ be double-framed soft sets 
over a common universe $U$. Then $\langle(\tilde{\alpha}, \tilde{\beta}) ; A\rangle$ is called a doubleframed soft subset $[10 \mid$ of $\langle(\tilde{f}, \tilde{g}) ; B\rangle$, denoted by

$$
\langle(\tilde{\alpha}, \tilde{\beta}) ; A\rangle \tilde{\subseteq}\langle(\tilde{f}, \tilde{g}) ; B\rangle,
$$

if (i) $A \subseteq B$,

(ii) $(\forall e \in A)$

$\left(\begin{array}{l}\tilde{\alpha}(e) \text { and } \tilde{f}(e) \text { are identical approximations, } \\ \tilde{\beta}(e) \text { and } \tilde{g}(e) \text { are identical approximations. }\end{array}\right)$

Theorem 3.10. Let $\langle(\tilde{\alpha}, \tilde{\beta}) ; A\rangle$ be a double-framed soft subset of a double-framed soft set $\langle(\tilde{f}, \tilde{g}) ; B\rangle$. If $\langle(\tilde{f}, \tilde{g}) ; B\rangle$ is a double-framed soft deductive system of $B$ over $U$, then so is $\langle(\tilde{\alpha}, \tilde{\beta}) ; A\rangle$.

Proof. Since $x \in A$ for all $x \in B$, we have $\tilde{\alpha}(x)=\tilde{f}(x) \subseteq$ $\tilde{f}(0)=\tilde{\alpha}(0)$ and $\tilde{\beta}(x)=\tilde{g}(x) \supseteq \tilde{g}(0)=\tilde{\beta}(0)$. Let $x, y \in A$.

Then $x, y \in B$, and so

$$
\begin{aligned}
& \tilde{\alpha}(x-y) \cap \tilde{\alpha}(y)=\tilde{f}(x-y) \cap \tilde{f}(y) \subseteq \tilde{f}(x)=\tilde{\alpha}(x), \\
& \tilde{\beta}(x-y) \cup \tilde{\beta}(y)=\tilde{g}(x-y) \cup \tilde{g}(y) \supseteq \tilde{g}(x)=\tilde{\beta}(x) .
\end{aligned}
$$

Hence $\langle(\tilde{\alpha}, \tilde{\beta}) ; A\rangle$ is a double-framed soft deductive system of $A$ over $U$.

Theorem 3.11. Let $\langle(\tilde{\alpha}, \tilde{\beta}) ; X\rangle$ be a double-framed soft of $X$ over $U$. Then the set

$$
\mathcal{D}(0):=\{x \in X \mid \tilde{\alpha}(x)=\tilde{\alpha}(0), \tilde{\beta}(x)=\tilde{\beta}(0)\}
$$

is a deductive system of $X$.

Proof. Obviously $0 \in \mathcal{D}(0)$. Let $x, y \in X$ be such that $x-y \in$ $\mathcal{D}(0)$ and $y \in \mathcal{D}(0)$. Then $\tilde{\alpha}(x-y)=\tilde{\alpha}(0)=\tilde{\alpha}(y)$ and $\tilde{\beta}(x-y)=\tilde{\beta}(0)=\tilde{\beta}(y)$. This implies that $\tilde{\alpha}(x) \supseteq \tilde{\alpha}(x-$ $y) \cap \tilde{\alpha}(y)=\tilde{\alpha}(0)$ and $\tilde{\beta}(x) \subseteq \tilde{\beta}(x-y) \cup \tilde{\beta}(y)=\tilde{\beta}(0)$ by (c2). Since $\tilde{\alpha}(0) \supseteq \tilde{\alpha}(x)$ and $\tilde{\beta}(0) \subseteq \tilde{\beta}(x)$ for all $x \in X$, it follows that $\tilde{\alpha}(x)=\tilde{\alpha}(0)$ and $\tilde{\beta}(x)=\tilde{\beta}(0)$ so that $x \in \mathcal{D}(0)$. Therefore $\mathcal{D}(0)$ is a deductive system of $X$.

For two double-framed soft sets $\langle(\tilde{\alpha}, \tilde{\beta}) ; A\rangle$ and $\langle(\tilde{f}, \tilde{g}) ; A\rangle$ over $U$, the double-framed soft int-uni set $[10]$ of $\langle(\tilde{\alpha}, \tilde{\beta}) ; A\rangle$ and $\langle(\tilde{f}, \tilde{g}) ; A\rangle$ is defined to be a double-framed soft set

$$
\langle(\tilde{\alpha} \otimes \tilde{f}, \tilde{\beta} \oplus \tilde{g}) ; A\rangle,
$$

where

$$
\begin{gathered}
\tilde{\alpha} \otimes \tilde{f}: A \rightarrow \mathscr{P}(U), x \mapsto \tilde{\alpha}(x) \cap \tilde{f}(x), \\
\tilde{\beta} \oplus \tilde{g}: A \rightarrow \mathscr{P}(U), x \mapsto \tilde{\beta}(x) \cup \tilde{g}(x) .
\end{gathered}
$$

It is denoted by $\langle(\tilde{\alpha}, \tilde{\beta}) ; A\rangle \sqcap\langle(\tilde{f}, \tilde{g}) ; A\rangle=\langle(\tilde{\alpha} \otimes \tilde{f}, \tilde{\beta} \oplus \tilde{g})$; A).

Theorem 3.12. The double-framed soft int-uni set of two double-framed soft deductive system $\langle(\tilde{\alpha}, \tilde{\beta}) ; A\rangle$ and $\langle(\tilde{f}, \tilde{g}$; $A\rangle$ of $A$ over $U$ is a double-framed soft deductive system of $A$ over $U$.

Proof. For all $x \in A$, we have $(\tilde{\alpha} \otimes \tilde{f})(x)=\tilde{\alpha}(x) \cap \tilde{f}(x) \subseteq$ $\tilde{\alpha}(0) \cap \tilde{f}(0)=(\tilde{\alpha} \otimes \tilde{f})(0)$ and $(\tilde{\beta} \oplus \tilde{g})(0)=\tilde{\beta}(0) \cup \tilde{g}(0) \subseteq$ $\tilde{\beta}(x) \cup \tilde{g}(x)=(\tilde{\beta} \oplus \tilde{g})(x)$. Let $x, y \in A$. Then $x, y \in B$, and so

$$
\begin{aligned}
& (\tilde{\alpha} \otimes \tilde{f})(x-y) \cap(\tilde{\alpha} \otimes \tilde{f})(y) \\
& =(\tilde{\alpha}(x-y) \cap \tilde{f}(x-y)) \cap(\tilde{\alpha}(y) \cap \tilde{f}(y)) \\
& =\tilde{\alpha}(x-y) \cap \tilde{\alpha}(y)) \cap(\tilde{f}(x-y) \cap \tilde{f}(y)) \\
& \subseteq \tilde{\alpha}(x) \cap \tilde{f}(x)=(\tilde{\alpha} \otimes \tilde{f})(x),
\end{aligned}
$$

and

$$
\begin{aligned}
& (\tilde{\beta} \oplus \tilde{g})(x-y) \cup(\tilde{\beta} \oplus \tilde{g})(y) \\
& =(\tilde{\beta}(x-y) \cup \tilde{g}(x-y)) \cup(\tilde{\beta}(y) \cup \tilde{g}(y)) \\
& =(\tilde{\beta}(x-y) \cup \tilde{\beta}(y)) \cup(\tilde{g}(x-y) \cup \tilde{g}(y)) \\
& \supseteq \tilde{\beta}(x) \cup \tilde{g}(x)=(\tilde{\beta} \oplus \tilde{g})(x),
\end{aligned}
$$

Hence $\langle(\tilde{\alpha} \otimes \tilde{f}, \tilde{\beta} \oplus \tilde{g}) ; A\rangle$ is a double-framed soft deductive system of $A$ over $U$.

\section{Conflict of Interest}

No potential conflict of interest relevant to this article was reported.

\section{References}

[1] L. A. Zadeh, "Fuzzy sets," Information and Control, vol. 8, no. 3, pp. 338-353, 1965. https://doi.org/10.1016/S00199958(65)90241-X

[2] D. Molodtsov, "Soft set theory: first results," Computers \& Mathematics with Applications, vol. 37, no. 5, pp. 19-31, 1999. https://doi.org/10.1016/S0898-1221(99)00056-5

[3] P. K. Maji, A. R. Roy, and R. Biswas, "An application of soft sets in a decision making problem," Computers 
\& Mathematics with Applications, vol. 44, no. 8-9, pp. 1077-1083, 2002.

[4] P. K. Maji, R. Biswas, and A. R. Roy, "Soft set theory," Computers \& Mathematics with Applications, vol. 45, no. 4-5, pp. 555-562, 2003.

[5] H. Aktas and N. Cagman, "Soft sets and soft groups," Information Sciences, vol. 177, no. 13, pp. 2726-2735, 2007. https://doi.org/10.1016/j.ins.2006.12.008

[6] Y. B. Jun and C. H. Park, "Applications of soft sets in ideal theory of BCK/BCI-algebras," Information Sciences, vol. 178, no. 11, pp. 2466-2475, 2008. https://doi.org/10. 1016/j.ins.2008.01.017

[7] H. S. Al-Saadi and W. K. Min, "On soft generalized closed sets in a soft topological space with a soft weak structure," International Journal of Fuzzy Logic and Intelligent Systems, vol. 17, no. 4, pp. 323-328, 2017. http://dx.doi.org/10.5391/IJFIS.2017.17.4.323

[8] U. Acar, F. Koyuncu, and B. Tanay, "Soft sets and soft rings," Computers \& Mathematics with Applications, vol. 59, no. 11, pp. 3458-3463, 2010. https://doi.org/10.1016/j. camwa.2010.03.034

[9] Y. B. Jun, K. J. Lee, and C. H. Park, "Soft set theory applied to ideals in d-algebras," Computers \& Mathematics with Applications, vol. 57, no. 3, pp. 367-378, 2009. https://doi.org/10.1016/j.camwa.2008.11.002

[10] Y. B. Jun and S. S. Ahn, "Double-framed soft sets with applications in BCK/BCI-algebras," Journal of Applied Mathematics, vol. 2012, article ID. 178159, 2012. http: //dx.doi.org/10.1155/2012/178159

[11] Y. B. Jun, K.J.Lee, C. H. Park, and E. H. Roh, "Filters of residuated lattices based on soft set theory," Communications of the Korean Mathematical Society, vol. 30, no. 3, pp. 155-168, 2015. http://dx.doi.org/10.4134/CKMS. 2015.30.3.155

[12] G. Muhiuddin, C. H. Park, and Y. B. Jun, "Relations between regular uni-soft filters and uni-soft MV-filters in residuated lattices," Kyungpook Mathematical Journal, vol. 57, no. 1, pp. 85-97, 2017. https://doi.org/10.5666/ kmj.2017.57.1.85
[13] C. H. Park, Y. B. Jun, and M. A. Ozturk, "Soft WSalgebras," Communications of the Korean Mathematical Society, vol. 23, no. 3, pp. 313-324, 2008. https: //doi.org/10.5391/IJFIS.2011.11.2.071

[14] J. H. Park, Y. C. Kwun, and M. J. Son, “A generalized intuitionistic fuzzy soft set theoretic approach to decision making problems," International Journal of Fuzzy Logic and Intelligent Systems, vol. 11, no. 2, pp. 71-76, 2011. https://doi.org/10.5391/IJFIS.2011.11.2.071

[15] J. H. Park, "Operations on generalized intuitionistic fuzzy soft sets," International Journal of Fuzzy Logic and Intelligent Systems, vol. 11, no. 3, pp. 184-189, 2011. https://doi.org/10.5391/IJFIS.2011.11.3.184

[16] B. M. Schein, "Difference semigroups," Comm. in Alge bra, vol. 20, no. 8, pp. 2153-2169, 1992. https://doi.org/ $10.1080 / 00927879208824453$

[17] J. C. Abbott, Sets, lattices, and Boolean algebras. Boston, MA: Allyn and Bacon, 1969.

[18] Y. B. Jun and H. S. Kim, "On ideals in subtraction algebras," Scientiae Mathematicae Japonicae, vol. 65, no. 1, pp. 129-134, 2007.

[19] Y. B. Jun, Y. H. Kim, and K. A. Oh, "Subtraction algebras with additional conditions," Communications of the Korean Mathematical Society, vol. 22, no. 1, pp. 1-7, 2007.

[20] J. Zhan and Y. B. Jun, "Soft BL-algebras based on fuzzy sets," Computers \& Mathematics with Applications, vol 59, no. 6, pp. 2037-2046, 2010. https://doi.org/10.1016/j. camwa.2009.12.008

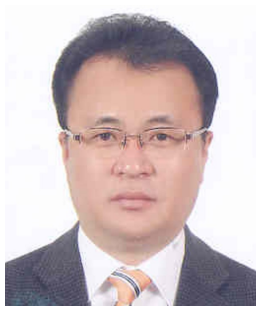

Chul Hwan Park is currently a faculty member at the School of Mechanical Engineering, Ulsan College, Ulsan, Korea since 2011. He has served as an expert reviewer for several journal (SCI, SCIE, SCOPUS, KCI) From 1997 to 1998, he was a visiting researcher at the Institute of Basic Science, The Kyungpook National Universty, Korea (supported by KOSEF) and worked for year (2005-2009) as a professor (full time lecture) at the Department of Mathematics, University of Ulsan, Ulsan, Korea. His research interests focus on the theory of fuzzy algebraic structure, quantum structure and commutative algebra.

E-mail: chpark2@uc.ac.kr, skyrosemary@gmail.com 